Hautarzt 2021 · 72:984-991

https://doi.org/10.1007/s00105-021-04871-9

Angenommen: 7. Juli 2021

Online publiziert: 20. August 2021

(c) Der/die Autor(en) 2021

\section{Behandlung der Psoriasis mit Secukinumab}

\author{
Praxisrelevante Hinweise
}

\author{
Andreas Körber' $\cdot$ Matthias Augustin ${ }^{2} \cdot$ Frank Behrens $^{3} \cdot$ Sascha Gerdes $^{4} \cdot$ Ralph von \\ Kiedrowski ${ }^{5} \cdot$ Knut Schäkel $^{6} \cdot$ Michael Sticherling $^{7} \cdot$ Dagmar Wilsmann-Theis $^{8}$. \\ Johannes Wohlrab ${ }^{9} \cdot$ Jan-Christoph Simon ${ }^{10}$ \\ ' Dermatologische Privatpraxis, Essen, Deutschland; ${ }^{2}$ Institut für Versorgungsforschung in der \\ Dermatologie und bei Pflegeberufen (IVDP), Universitätsklinikum Hamburg-Eppendorf, Hamburg, \\ Deutschland; ${ }^{3}$ Rheumatologie, Medizinische Klinik 2, Universitätsklinikum Goethe-Universität, Frankfurt \\ am Main, Deutschland; ${ }^{4}$ Psoriasis-Zentrum, Klinik für Dermatologie, Venerologie und Allergologie, \\ Universitätsklinikum Schleswig-Holstein Campus Kiel, Kiel, Deutschland; ${ }^{5}$ Dermatologische Spezialpraxis \\ für chronisch-entzündliche System-Dermatosen, Dermato-Onkologie und Allergologie, Selters \\ (Westerwald), Deutschland; ${ }^{6}$ Hautklinik, Universitätsklinikum Heidelberg, Heidelberg, Deutschland; \\ ${ }^{7}$ Hautklinik und Psoriasiszentrum, Universitätsklinikum Erlangen, Deutsches Zentrum Immuntherapie, \\ Erlangen, Deutschland; ${ }^{8}$ Klinik und Poliklinik für Dermatologie und Allergologie, Universitätsklinikum \\ Bonn, Bonn, Deutschland; ' ${ }^{2}$ Universitätsklinik für Dermatologie und Venerologie, Martin-Luther- \\ Universität Halle-Wittenberg, Halle, Deutschland; ${ }^{10} \mathrm{Klinik}$ und Poliklinik für Dermatologie, Venerologie \\ und Allergologie, Universitätsklinikum Leipzig AöR, Leipzig, Deutschland
}

Hintergrund: Die mittelschwere bis schwere Psoriasis vulgaris kann wirksam mit immunmodulierenden Biologika wie dem Interleukin-17A-Inhibitor Secukinumab behandelt werden. In der Praxis stellt sich jedoch oft die Frage nach dem Vorgehen in besonderen Situationen, beispielsweise bei Infektionen, Komorbidität, Schwangerschaft oder operativen Eingriffen.

Ziel der Arbeit: In diesem Konsensdokument deutscher Psoriasisexperten sollen in Ergänzung zu den aktuellen Leitlinien häufige Fragen aus dem Therapiealltag zur Behandlung der Psoriasis mit Secukinumab beantwortet werden.

Methoden: In einem virtuellen Expertentreffen im Mai 2020 wurden auf Grundlage von Erfahrungen der Teilnehmer und aktueller Literatur praxisrelevante Aspekte der Behandlung der Psoriasis erörtert. Darauf basierend wurde ein Konsensdokument verfasst.

Ergebnisse: Die vorliegende Arbeit bietet praktische Hinweise zur Anamnese einschließlich der Erfassung von Vortherapien, Schweregrad der Psoriasis und Begleiterkrankungen vor Beginn einer Therapie mit Secukinumab. Ferner wird auf das Vorgehen bei Impfungen, Infektionen, operativen Eingriffen, Sondermanifestationen der Psoriasis und Komorbiditäten einschließlich vorbestehenden Autoimmunerkrankungen und Tumorerkrankungen unter Therapie mit Secukinumab eingegangen. Auch Fragen zur Familienplanung und zu gesundheitspolitischen Regularien werden diskutiert.

Diskussion: Die in diesem Konsensdokument zusammengefassten unterstützenden Empfehlungen zur Behandlung der Psoriasis mit Secukinumab sollen dazu beitragen, für die Patienten eine optimale Therapie zu erreichen und ihre Lebensqualität zu verbessern.

\section{Schlüsselwörter}

Infektionen · Komorbidität · Perioperatives Management · Biologika · Besondere Situationen 
Die mittelschwere bis schwere Psoriasis vulgaris kann wirksam mit immunmodulierenden Biologika wie dem Interleukin17A-Inhibitor Secukinumab behandelt werden [18, 19]. Die Indikationsstellung berücksichtigt die individuellen gesundheitlichen Voraussetzungen der Patienten wie akute oder chronische Infektionen, Komorbiditäten einschließlich Autoimmunund Tumorerkrankungen sowie Situationen wie Schwangerschaft oder geplante operative Eingriffe. In einem virtuellen Treffen deutscher Psoriasisexperten aus Klinik und Praxis im Mai 2020 wurden diese und weitere praxisrelevante Aspekte in Bezug auf die Therapie mit Secukinumab erörtert. Die differenzialtherapeutische Wahl des Medikaments bei Vorliegen verschiedener Therapieoptionen wurde hierbei nicht diskutiert. Auf Grundlage der Erfahrung der Experten sowie des Literaturhintergrunds bieten die folgenden Empfehlungen in Ergänzung zu aktuellen Leitlinien eine Hilfe bei Anamnese, Therapie und Management der Psoriasis.

\section{Anamnese und Laborkontrolle}

Voraussetzungen der Indikationsstellung für eine Therapie der Psoriasis vulgaris mit Secukinumab sind:

- Vorliegen einer Psoriasis vulgaris (ICD10 L40.0); auch das Vorliegen einer Psoriasisarthritis kann eine Indikation zur Therapie mit Secukinumab sein, ist aber nicht Gegenstand des vorliegenden Manuskriptes,

- Ermittlung des Schweregrads (mittelschwer bis schwer: ICD10 L40.70!) anhand Psoriasis Area and Severity Index (PASI) und/oder Body Surface Area $(B S A)>10$ sowie Dermatology Life Quality Index (DLQI) > 10,

- spezielle klinische Situationen (wie besondere Lokalisation oder starker Nagelbefall) stellen Upgrade-Kriterien bei insgesamt geringem Hautbefall dar [17],

- Ausschluss von Kontraindikationen wie Schwangerschaft, klinisch relevanten aktiven Infektionen (wie aktiver Tuberkulose) oder chronisch entzündlichen Darmerkrankungen (relative Kontraindikation),

- Abklärung chronischer oder rezidivierender Infektionen; bei Vorliegen sollte Secukinumab nur mit Vorsicht angewendet werden,

- Ausschluss einer Überempfindlichkeit gegen den Wirkstoff oder einen weiteren Bestandteil des Medikaments.

Darüber hinaus sollen gemäß Leitlinien Krankheitsbeginn, -dauer und -schweregradverlauf, Familienanamnese, Hinweise auf eine Psoriasisarthritis (PsA), individuelle Triggerfaktoren, Vortherapien, Impfstatus, Infektions- und Tumoranamnese, Komorbidität und Familienplanung erfasst werden [18, 19]. Insbesondere in Bezug auf die letzten 5 Punkte ist ggf. vor Behandlungsbeginn eine besondere Patientenaufklärung erforderlich. Die Anamnese der Vortherapien sollte strukturiert erfolgen und möglichst zu jedem Wirkstoff Dosis, Zeitpunkt von Therapiebeginn und -ende sowie Abbruchgrund erfassen. Vor Beginn der Therapie mit Secukinumab sollten Begleiterkrankungen (z.B. chronisch entzündliche Darmerkrankungen) sowie chronische oder rezidivierende Infektionen in der Vorgeschichte (u.a. Tuberkulose, Hepatitis B) erfragt und aktive Infektionen sowie eine Schwangerschaft ausgeschlossen werden. Spezifische Laboruntersuchungen während der Therapie mit Secukinumab sind gemäß Fachinformation nicht erforderlich [10]. Vor Therapiebeginn, 4 und 12 Wochen danach sowie alle 3 Monate während des weiteren Therapieverlaufs empfiehlt die aktuelle Psoriasisleitlinie dennoch ein Differenzialblutbild und die Bestimmung der Leberwerte.

ZurErmittlung des Komorbiditätsrisikos beim ersten Patientenkontakt steht eine Checkliste mit Handlungsempfehlungen zur Verfügung (Supplement) [32], die sich am bundesweiten Konsens zur Früherkennung von Komorbidität orientieren [20]. Alle 3 bis 6 Monate sollten der PASI und die Lebensqualität mittels DLQI erfasst werden. Insbesondere nach der Induktionsphase mit Secukinumab (ca. 12 Wochen) sollten diese Parameter genutzt werden, um ein ausreichendes Therapieansprechen zu prüfen.

\section{Wechselwirkungen}

Biologika wie Secukinumab werden nicht über das Enzymsystem der Leber meta- bolisiert und benötigen für ihre Clearance keine Transporter. Von einer direkten Wechselwirkung mit Small-Molecule-Medikamenten einschließlich Methotrexat (MTX) und/oder Kortikosteroiden ist nicht auszugehen. Eine Kombination von Secukinumab mit anderen immunmodulierenden Biologika, die ein erhöhtes Infektionsrisiko zur Folge haben, wird aufgrund unzureichender Studienlage nicht empfohlen. Positive Einzelfallberichte zur Kombination mit Apremilast liegen vor [23]. Falls eine Kombination verschiedener Biologika im Einzelfall notwendig erscheint, sollte dies beispielsweise im Rahmen eines interdisziplinären Entzündungsboards abgestimmt werden.

\section{Impfungen}

Patienten unter immunmodulierender Therapie sollten gemäß Empfehlungen der Ständigen Impfkommission (STIKO) gegen Influenza, Pneumokokken und Meningokokken geimpft sein [25]. Die Autoren empfehlen darüber hinaus eine Impfung gegen Haemophilus influenzae b (Hib). Impfungen mit inaktivierten und Totimpfstoffen sind unter SecukinumabTherapie ohne Weiteres möglich und sollten im Idealfall in der Mitte eines Behandlungsintervalls erfolgen. Studien haben gezeigt, dass Secukinumab die Impfantwort auf eine Influenza- oder Meningokokkenimpfung nicht negativ beeinflusst $[5,11]$. Im Gegensatz dazu ist eine Impfung mit Lebendimpfstoff unter immunmodulierender und potenziell die Infektionsabwehr beeinflussender Biologikatherapie grundsätzlich kontraindiziert (Übersicht in - Tab. 1). Lebendimpfungen sollten mindestens 4 Wochen vor Therapiebeginn erfolgt sein oder frühestens 2 Monate nach Therapieunterbrechung bzw. -ende durchgeführt werden [30]. Die Unterscheidung zwischen Lebendund Tot- bzw. inaktivierten Impfstoffen ist auch für neu entwickelte Impfstoffe beispielsweise gegen COVID-19 zu berücksichtigen. RNA(Ribonukleinsäure)und Vektor-basierte Impfstoffe können Totimpfstoffen gleichgesetzt werden. Die Therapie mit Secukinumab stellt daher keine Kontraindikation für die Impfung gegen COVID-19 dar. 
Tab. 1 Eignung verschiedener Impfstoffe zur Impfung unter Therapie mit Secukinumab. (Mod. nach [30])

\begin{tabular}{|c|c|c|}
\hline Impfung gegen & Impfstofftyp & Impfung während Therapie \\
\hline Haemophilus influenzae b (Hib) & Totimpfstoff & Möglich ${ }^{\mathrm{a}}$ \\
\hline Meningokokken & Totimpfstoff & Möglich $^{\mathrm{a}}$ \\
\hline Pneumokokken & Totimpfstoff & Möglich ${ }^{\mathrm{a}}$ \\
\hline Diphtherie-Tetanus-Pertussis & Totimpfstoff & Möglich \\
\hline Hepatitis $B$ & Totimpfstoff & Möglich ${ }^{\mathrm{a}}$ \\
\hline \multirow[t]{2}{*}{ Herpes Zoster } & Totimpfstoff & Möglich ${ }^{\mathrm{a}}$ \\
\hline & Lebendimpfstoff & Kontraindiziert \\
\hline Humane Papillomviren (HPV) & Totimpfstoff & Möglich ${ }^{\mathrm{a}}$ \\
\hline Frühsommermeningoenzephalitis (FSME) & Totimpfstoff & Möglich ${ }^{\mathrm{a}}$ \\
\hline \multirow[t]{2}{*}{ Influenza } & Totimpfstoff & Möglich ${ }^{\mathrm{a}}$ \\
\hline & Lebendimpfstoff & Kontraindiziert \\
\hline Masern-Mumps-Röteln (MMR) & Lebendimpfstoff & Kontraindiziert \\
\hline Varizellen & Lebendimpfstoff & Kontraindiziert \\
\hline \multicolumn{3}{|l|}{ Reiseimpfungen } \\
\hline Cholera & Totimpfstoff & Möglich $^{\mathrm{a}}$ \\
\hline Gelbfieber & Lebendimpfstoff & Kontraindiziert \\
\hline Hepatitis A & Totimpfstoff & Möglich $^{\mathrm{a}}$ \\
\hline Japanische Enzephalitis & Totimpfstoff & Möglich $^{\mathrm{a}}$ \\
\hline \multirow[t]{2}{*}{ Typhus } & Totimpfstoff & Möglich ${ }^{\mathrm{a}}$ \\
\hline & Lebendimpfstoff & Kontraindiziert \\
\hline
\end{tabular}

\section{Infektionen}

Vor Beginn einer Therapie mit Secukinumab sollten eine Tuberkulose und mögliche Infektionen mit Hepatitis B oder C sowie bei Vorliegen entsprechender Risikofaktoren auch HIV(,human immunodeficiency virus")- oder weitere chronische Infektionen abgeklärt werden. Bei klinisch relevanten, aktiven Infektionen besteht eine Kontraindikation. Bei Patienten mit einer chronischen Infektion oder einer rezidivierenden Infektion in der Vorgeschichte sollte Secukinumab mit Vorsicht angewendet werden. Das Vorliegen einer Tuberkulose sollte vor Therapiebeginn mittels Thoraxröntgenaufnahme und IGRA(Interferon Gamma Release Assay)-Test beurteilt werden. Bei Nachweis einer latenten Tuberkulose sollte erwogen werden, 1 Monat vor Therapiebeginn eine Chemoprävention mittels Isoniazid (INH) zu beginnen und diesefürinsgesamt 9 Monate fortzuführen. Alternativ ist eine Kombinationstherapie mit INH und Rifampicin (RMP) für insgesamt 3 bis 4 Monate bzw. bei INH-Resistenz oder -Unverträglichkeit eine RMP-Monotherapie über 4 Monate möglich [24].
Unter Behandlung mit Secukinumab können vermehrt leichte oder mittelschwere Infektionen der oberen Atemwege sowie Kandidosen auftreten, die jedoch in der Regel keinen Behandlungsabbruch erfordern und auf Standardbehandlung ansprechen [18]. Von einer klinisch relevanten Steigerung des Risikos schwerer bakterieller Infektionen unter Secukinumab wird nicht ausgegangen. Bei Auftreten von Infektionen, insbesondere bei leichten akuten Infektionen, besteht kein grundsätzlich erhöhter Bedarf für eine antibiotische Therapie. Die Entscheidung über Einleitung einer antibiotischen Therapie sollte anhand der individuellen Situation des Patienten unabhängig von der Secukinumab-Therapie getroffen werden.

Bei akuten fieberhaften Infektionen sollte die Therapie pausiert werden. Abhängig von Allgemeinzustand und individueller Situation des Patienten kann in Absprache mit dem behandelnden Arzt frühestens $48 \mathrm{~h}$ nach Fieberfreiheit eine Fortsetzung der Therapie erwogen werden. Der Zeitpunkt der ersten Gabe nach Fieberfreiheit kann als Beginn eines neuen Behandlungsintervalls betrachtet werden.

Die Inzidenz viraler Infektionen der Atemwege und grippaler Infekte war in einer aktuellen Datenanalyse unter Secukinumab gegenüber Placebo nicht erhöht [7]. Bezüglich SARS-CoV-2 empfiehlt die Expertengruppe von BVDD (Berufsverband der Deutschen Dermatologen e. V.) und DDG (Deutsche Dermatologische Gesellschaft e. V.), bei positiv auf das Virus getesteten Patienten ein Herauszögern der Secukinumab-Therapie für die Dauer der mittleren Inkubationszeit von SARSCoV-2 zu erwägen. Beim Auftreten von COVID-19-Symptomen sollte die Therapie bis zu deren Abklingen pausiert werden [2].

\section{Perioperatives Management}

Je nach Art und Umfang eines operativen Eingriffs kann eine Biologikatherapie mit einem erhöhten Infektionsrisiko assoziiert sein. Hierzu liegen allerdings nur wenige Studiendaten und keine deutschen Leitlinienempfehlungen vor [18]. Die Entscheidung über eine Unterbrechung der Therapie mit Secukinumab vor operativen Eingriffen sollte neben patientenbezogenen Parametern wie Alter, Begleiterkrankungen und ggf. bereits bestehenden Infektionen auch das mit dem Eingriff verbundene Infektionsrisiko sowie die zeitliche Planbarkeit des Eingriffs berücksichtigen. Als Hochrisikoeingriffe in diesem Kontext sind insbesondere Notfalloperationen sowie Eingriffe an Herz, Thorax und Gefäßen zu sehen, während dermatologische Operationen in der Regel als Eingriffe mit mittlerem oder eher niedrigem Risiko einzustufen sind (• Tab. 2; [6]).

Bei elektiven Eingriffen mit mittlerem bis hohem Risiko wird eine Unterbrechung der Secukinumab-Therapie in einem zeitlichen Abstand von etwa 3 Halbwertszeiten (12 Wochen) vor dem Eingriff empfohlen. Bei Eingriffen mit geringem Risiko kann hingegen meist auf eine Therapieunterbrechung verzichtet werden. Notfalloperationen sind jederzeit ohne besondere vorhergehende Maßnahmen in Bezug auf die Secukinumab-Therapie durchführbar, wobei das möglicherweise erhöhte Infektionsrisiko beachtet werden muss. 


\begin{tabular}{|l|l|}
\hline $\begin{array}{l}\text { Tab. } 2 \\
\text { fe unter Therapie mit immunmodulierenden } \\
\text { Biologika. (Mod. nach [6]) }\end{array}$ \\
\hline Risiko & Art des Eingriffs \\
\hline Hoch & Notfalloperation \\
\cline { 2 - 2 } & Größere Thoraxoperation \\
\cline { 2 - 2 } & Herzchirurgischer Eingriff \\
\cline { 2 - 2 } & $\begin{array}{l}\text { Eingriff an der Aorta oder grö- } \\
\text { Berer gefäßchirurgischer Eingriff }\end{array}$ \\
\cline { 2 - 2 } & Peripherer arterieller Eingriff \\
\cline { 2 - 2 } & Operationszeit von über 4 $\mathrm{h}$ \\
\hline Moderat & Orthopädischer Eingriff \\
\cline { 2 - 2 } & Urologischer Eingriff \\
\cline { 2 - 2 } & $\begin{array}{l}\text { Unkomplizierter Eingriff an } \\
\text { Thorax oder Abdomen }\end{array}$ \\
\cline { 2 - 2 } & $\begin{array}{l}\text { Unkomplizierter Eingriff an Kopf } \\
\text { oder Hals }\end{array}$ \\
\cline { 2 - 3 } & Karotisendarteriektomie \\
\hline & Eingriff an der Prostata \\
\hline Gering & Endoskopie \\
\hline & Bronchoskopie \\
\hline & Hysteroskopie \\
\hline & Zystoskopie \\
\hline & Dermatochirurgische Eingriffe \\
\hline Brustbiopsie bzw. -exzision \\
\hline & Ophthalmologischer Eingriff \\
\hline Hautbiopsien \\
\hline
\end{tabular}

Eine kleinere retrospektive Studie zum Risiko postoperativer Komplikationen unter Biologikatherapie bei Psoriasis oder PsA kam zu dem Schluss, dass eine Therapieunterbrechung keinen Unterschied in Bezug auf Infektionsrisiko oder Wundheilungsstörungen mit sich bringt, jedoch signifikant mit einer Symptomverschlechterung der Psoriasis bzw. PsA assoziiert ist [4]. Die möglichen Vor- und Nachteile einer Therapieunterbrechung sind daher abhängig von Eingriff und individueller Patientensituation gegeneinander abzuwägen.

\section{Psoriasisarthritis und Sonder- manifestationen}

Die Therapie eines Psoriasispatienten mit gleichzeitiger PsA sollte beiden Krankheitsbildern gerecht werden. Bei Patienten mit PsA und gleichzeitiger mittelschwerer bis schwerer Plaquepsoriasis oder solchen, die auf TNF(Tumor-Nekrose-Faktor)-Inhibitoren unzureichend angesprochen haben, beträgt die Secukinumab-Dosis $300 \mathrm{mg}$ [10]. In der klinischen Phase-III-Studie zum Vergleich von Secukinumab mit dem
TNF-Blocker Adalimumab bei Patienten mit PsA wurde gezeigt, dass Secukinumab bei Patienten mit begleitender Psoriasis vulgaris (BSA $\geq 3$ ) zu einem besseren PASI-100-Ansprechen führte $(p=0,0007)$, obwohl die Studie in Bezug auf die PsA ihren primären Endpunkt einer signifikant überlegenen Wirksamkeit knapp verfehlte. Secukinumab war zudem mit einer höheren Therapieadhärenz verbunden als Adalimumab [15].

$\mathrm{Zu}$ den Sondermanifestationen der Psoriasis vulgaris gehören Nagel-, Kopfhaut-, genitale und palmoplantare Psoriasis. Die signifikante Wirksamkeit von Secukinumab konnte in jeweils randomisierten, doppelblinden, placebokontrollierten Phase-III-Studien nachgewiesen werden [3, 12, 22]. Langzeitdaten aus der Extensionsphase der Studien zur Nagelund palmoplantaren Psoriasis belegen die andauernde Wirksamkeit in der Therapie der einzelnen Sondermanifestationen [13, 21].

Die generalisierte Psoriasis pustulosa (GPP) und die Pustulosis palmoplantaris (PPP) stellen eigenständige Krankheitsbilder dar (ICD10 L40.1 bzw. L40.3). Bei der GPP erreichten 83,3\% der Patienten in einer offenen Phase-III-Studie eine Symptomverbesserung innerhalb von 16 Wochen unter Secukinumab-Therapie [14]. Patienten mit PPP profitierten in einer randomisierten kontrollierten Studie von einer Behandlung mit Secukinumab in Form einer Verbesserung der Lebensqualität und des Palmoplantar Pustulosis Area and Severity Index (PPPASI) über 52 Wochen, obwohl der primäre Endpunkt der Studie in Form einer Verbesserung des PPPASI um $75 \%$ innerhalb von 16 Wochen nicht erreicht wurde [16]. Secukinumab ist nur in Japan für die Behandlung von GPP und PPP zugelassen.

\section{Familienplanung}

Eine Anwendung von Secukinumab während der Schwangerschaft ist gegenwärtig nicht zugelassen [10]. Es wird empfohlen, dass Frauen im gebärfähigen Alter während und für mindestens 12 Wochen (entsprechend 3 Halbwertszeiten) nach Ende der Secukinumab-Therapie eine zuverlässige Verhütungsmethode anwenden. Dennoch liegen Echtweltdaten zu ungeplanten Schwangerschaften unter Behandlung mit Secukinumab vor (Exposition im ersten Trimester). Es wurde keine höhere Anzahl von kongenitalen Malformationen oder Spontanaborten beobachtet als in der Gesamtbevölkerung [31]. Von einer diaplazentaren Übertragung von Immunglobulinen (einschließlich Secukinumab) an das ungeborene Kind ist während der ersten 2 Trimester nicht auszugehen. Die Autoren sehen es daher nicht als erforderlich an, eine unter Therapie mit Secukinumab eintretende Schwangerschaft abzubrechen. Eher kann eine engmaschige klinische und sonographisch gynäkologische Verlaufskontrolle der Patientin und des Fetus bis zur Entbindung erfolgen. Die Therapie sollte in diesem Fall jedoch ausgesetzt werden.

Die Anwendung von Secukinumab während der Stillzeit ist nicht zugelassen [10]. Die Wirkung von Secukinumab auf die Fertilität des Menschen wurde nicht untersucht. Tierexperimentelle Studien ergaben jedoch keine Hinweise auf nachteilige Wirkungen [10]. Daher wird nicht von negativen Auswirkungen auf die Fertilität ausgegangen. Bei Männern mit aktuellem Kinderwunsch ist eine Unterbrechung der Therapie nicht erforderlich.

\section{Gesundheitspolitische Regularien}

Zur Einhaltung gesundheitspolitischer Regularien in der Verschreibungspraxis ist es wichtig, Mindeststandards bei der Dokumentation zu wahren (ס Tab. 3). Eine Dokumentation ist dann ausreichend, wenn auch ein Außenstehender die Therapieentscheidung nachvollziehen kann. Bezogen auf die Therapie mit Secukinumab bedeutet dies vor Therapiebeginn eine entsprechend der ICD10Kodierung dokumentierte Diagnose sowohl der Psoriasis vulgaris (L40.0) als auch des erforderlichen Schweregrades (L40.70!) oder eines Upgrade-Kriteriums sowie eine Dokumentation der Scores, anhand derer der Schweregrad bestimmt wurde. Neben BSA und/oder PASI schließt dies auch ausdrücklich den DLQI ein [27]. Darüber hinaus sollten Begleiterkrankungen angemessen dokumentiert werden. Bei der Therapiebeschreibung ist auf eine dokumentierte Indikation zur Systemtherapie (Z51.88) zu achten. Zum Nachweis 
Tab. 3 Empfehlungen zur Dokumentation vor Beginn einer Therapie mit Secukinumab

Diagnose Psoriasis vulgaris (ICD10 L40.0)

Schweregrad mittelschwer bis schwer (ICD10 L40.70!)

Scores (BSA/PASI und DLQI)

Gegebenenfalls Upgrade-Kriterien benennen

Begleiterkrankungen (ICD10-kodiert) Indikation zur Systemtherapie (Z51.88)

Klar definiertes Therapieziel

Gegebenenfalls Vortherapie(n) und Grund für deren Beendigung (primäres oder sekundäres Versagen, Kontraindikationen, Unverträglichkeit)

Gegebenenfalls individuelle Faktoren

BSA Body Surface Area, PASI Psoriasis Area and Severity Index, DLQI Dermatology Life Quality Index

entsprechend Wirtschaftlichkeitsgebot (§ 12 SGB [Sozialgesetzbuch] V), dass die Behandlung wirtschaftlich, ausreichend, notwendig und zweckmäßig (WANZ) ist, sollte ein klar definiertes Therapieziel wie beispielsweise eine Verringerung des PASI entsprechend Leitlinie formuliert werden. Wichtig ist grundsätzlich auch die Dokumentation etwaiger Vortherapien und individueller Faktoren, sofern zutreffend. Die von einigen großen Krankenkassen geforderte Minimaldokumentation ist zudem im „Vertrag zur besonderen Versorgung in der Indikation Psoriasis" nach $\S$ 140a SGB V einzusehen. Dieser entspricht der Dokumentation im Deutschen Psoriasis-Register PsoBest, die als hinreichend für die Dokumentationspflicht in der Systemtherapie angesehen wird [1]. Eine angemessene Dokumentation lässt sich daher mit vertretbarem Aufwand umsetzen.

Die Prüfung der Wirtschaftlichkeit einer Verschreibung stellt sich bundesweit sehr heterogen dar. Bundeseinheitliche Vereinbarungen zwischen pharmazeutischen Unternehmen und dem Spitzenverband der Kassen stehen jedoch über regionalen Vereinbarungen. Eine anhand einer guten Dokumentation begründete Praxisbesonderheit bietet deshalb eine hohe Verordnungssicherheit.

\section{Vorbestehende Autoimmun- erkrankungen}

Es wird nicht davon ausgegangen, dass eine Therapie mit Secukinumab Autoimmunerkrankungen (u.a. Kollagenosen) auslösen oder verstärken kann. Bei Patienten mit multipler Sklerose (MS), systemischem Lupus erythematodes (SLE) oder Guillain-Barré-Syndrom (GBS) bestehen keine Einschränkungen. Erhöhte Spiegel antinukleärer Antikörper (ANA) vor Therapiebeginn stellen kein erhöhtes Risiko für den Einsatz von Secukinumab dar und sind keine Kontraindikation. Es wurden Fälle von Neuauftreten oder Exazerbationen von chronisch entzündlichen Darmerkrankungen unter Secukinumab berichtet. Bei einer bestehenden chronisch entzündlichen Darmerkrankung wird unabhängig von einer möglichen autoimmunen Ursache dieser Erkrankung eine Therapie mit Secukinumab nicht empfohlen. Wenn ein Patient Zeichen und Symptome einer chronisch entzündlichen Darmerkrankung entwickelt oder eine Exazerbation einer bestehenden chronisch entzündlichen Darmerkrankung auftritt, sollte die Behandlung mit Secukinumab beendet und geeignete medizinische Therapiemaßnahmen sollten eingeleitet werden.

\section{Wichtige Begleiterkrankungen}

Komorbidität kann bei der Psoriasis sehr unterschiedlich ausgeprägtsein (• Abb. 1). Konkrete Handlungsempfehlungen für bestimmte Begleiterkrankungen liegen vor [20]. Besonders häufig und erwähnenswert aufgrund ihrer gegenseitigen Beeinflussung sind das metabolische Syndrom, insbesondere Adipositas, sowie endotheliale Dysfunktion und Atherosklerose. Diese Begleiterkrankungen können durch systemische Entzündungsprozesse verstärkt werden, die im Zusammenhang mit der Psoriasis auftreten und durch Interleukin17A (IL-17A) gesteuert werden. Eine aktuelle Übersichtsarbeit fasst dies anschaulich zusammen [28]. Eine Anti-IL-17A-Therapie wie Secukinumab könnte sich daher auf diese Begleiterkrankungen positiv auswirken.

So zeigt eine aktuelle Beobachtungsstudie einen Zusammenhang zwischen einer Anti-IL-17A-Therapie und signifikan- tem Rückgang nicht kalzifizierter Koronarplaques um $12 \%$ nach 1 Jahr. Unter Anti-TNF- bzw. Anti-IL-12/23-Therapie wurde ein Rückgang um 5 bzw. $2 \%$ beobachtet [9]. In einer randomisierten klinischen Studie aus Deutschland wurde gezeigt, dass eine Anti-IL-17A-Therapie mit $300 \mathrm{mg}$ Secukinumab gegenüber Placebo die Endothelfunktion nach 52 Wochen verbessert $(p=0,0022)$, obwohl der primäre Endpunkt einer signifikanten Verbesserung nach 12 Wochen nicht erreicht wurde [29].

Auch auf den Glukosestoffwechsel und damit auf Übergewicht und Adipositas könnte sich eine IL-17A-Blockade durch die Interaktion der Signalwege von IL-17A und Insulinrezeptor positiv auswirken [28]. Bei vorbestehender Nieren- oder Leberinsuffizienz zeigten sich im klinischen Alltag bislang keine Signale für deren Verschlechterung unter SecukinumabTherapie.

\section{Tumorerkrankungen}

Eine aktuelle systematische Übersichtsarbeit zeigt bei Psoriasispatienten ein insgesamt leicht erhöhtes Risiko für Tumorerkrankungen, jedoch keinen Zusammenhang mit einer Therapie mit immunmodulierenden Biologika wie Secukinumab [26]. Auch in klinischen interventionellen und nichtinterventionellen Studien sowie Registerdaten wurden speziell zu Secukinumab keine erhöhten Raten an erstmanifesten malignen Tumoren im Vergleich zu Placebo oder aktiven Komparatoren beobachtet [8]. Bei einer bestehenden oder aus der Anamnese bekannten malignen Erkrankung ist eine Therapie mit Secukinumab entsprechend Zulassung und deutscher Leitlinie möglich [10, 18], während die europäische Leitlinie maligne und lymphoproliferative Erkrankungen als relative Kontraindikation einstuft [19]. Bei Patienten mit einer onkologischen Erkrankung, die sich noch in der Tumornachsorge befinden, sollte mit den behandelnden Kollegen eine Absprache bezüglich der Therapie mit Secukinumab erfolgen. Bei Patienten mit erhöhtem Risiko für maligne Tumoren bestehen keine prinzipiellen Einwände gegen den Einsatz von Secukinumab. Bei Patienten mit aktiver Tumorerkrankung sollte jedoch in konsequenter Zusammen- 


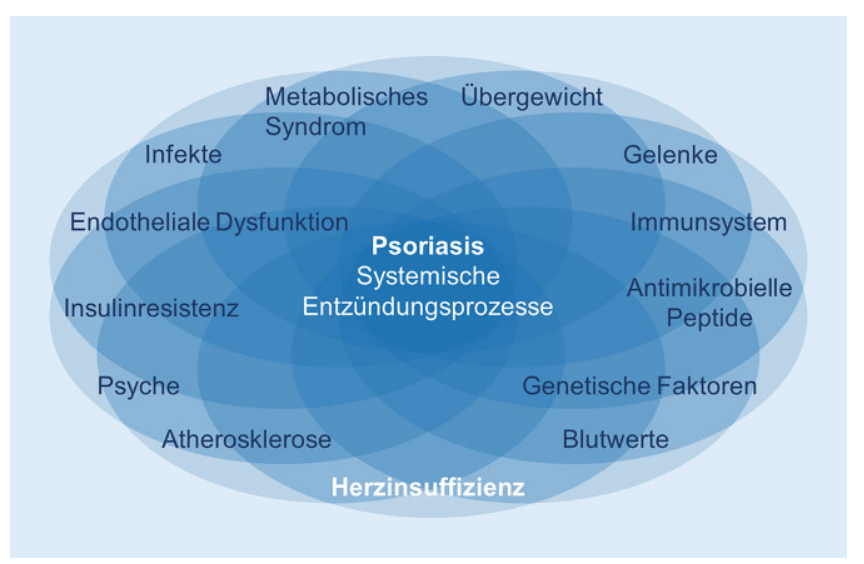

Abb. 1 ॥ Zusammenhang zwischen Psoriasis, kardiovaskulären Erkrankungen und metabolischem Syndrom. Viele Erkrankungen wie Psoriasis und Herzinsuffizienz beruhen auf systemischen Entzündungsprozessen und stehen im Zusammenhang mit weiteren Faktoren wie Adipositas und Depression. (Mod. nach [28])

arbeit mit den behandelnden Ärzten stets eine sorgfältige Nutzen-Risiko-Abwägung erfolgen, wobei für den Einsatz bei Patienten mit nichtmelanozytärem Hautkrebs oder Tumorerkrankungen mit geringem Risiko für Komplikationen im Zusammenhang mit der Psoriasis keine Einwände bestehen.

\section{Fazit für die Praxis}

- Vor Beginn einer Therapie mit Secukinumab sollten Vortherapien, Schweregrad der Psoriasis und Komorbiditäten erfasst und dokumentiert werden.

- Autoimmunerkrankungen sind keine Kontraindikation und bedeuten kein erhöhtes Risiko für den Einsatz von Secukinumab.

- Bei chronisch entzündlichen Darmerkrankungen wird eine Therapie mit Secukinumab nicht empfohlen. Bei kardiovaskulären Vorerkrankungen bestehen keine Einschränkungen.

- Impfungen mit inaktivierten und Totimpfstoffen sind unter Therapie möglich. Lebendimpfungen sind kontraindiziert.

- Bei fieberhaften Infektionen sollte die Therapie unterbrochen und frühestens $48 \mathrm{~h}$ nach Fieberfreiheit fortgesetzt werden.

- Bei operativen Eingriffen mit geringem Risiko ist meist keine Therapieunterbrechung nötig.

- Eine Anwendung während der Schwangerschaft ist nicht zugelassen. Der Abbruch einer unter Therapie eingetretenen Schwangerschaft ist nicht erforderlich.

- Es wurde kein Zusammenhang zwischen der Anwendung von Secukinumab und Tumorerkrankungen festgestellt. Bei vorbestehender Tumorerkrankung ist eine
Therapie in Absprache mit den behandelnden Ärzten ggf. möglich.

\section{Korrespondenzadresse}

\section{Prof. Dr. med. Jan-Christoph Simon}

Klinik und Poliklinik für Dermatologie, Venerologie und Allergologie, Universitätsklinikum Leipzig AöR

Philipp-Rosenthal-Str. 23, 04103 Leipzig,

Deutschland

Jan-Christoph.Simon@medizin.uni-leipzig.de

Danksagung. Die Autoren danken Dr. Philip Böhler (KW medipoint) für redaktionelle Unterstützung.

Förderung. Die redaktionelle Unterstützung wurde von der Novartis Pharma GmbH entsprechend der Leitlinie zur Good Publication Practice (GPP3) finanziert (http://www.ismpp.org/gpp3). Für die Inhalte sind ausschließlich die Autorinnen und Autoren verantwortlich.

Funding. Open Access funding enabled and organized by Projekt DEAL.

\section{Einhaltung ethischer Richtlinien}

Interessenkonflikt. A. Körber: Berater, Redner oder Teilnahme an klinischen Studien gesponsert durch AbbVie, Almirall, Amgen, Biogen, Boehringer Ingelheim Pharma, Bristol Myers Squibb, Celgene, JanssenCilag, Leo Pharma, Lilly, Merck Sharp \& Dohme Corp., Novartis, Pfizer und UCB. M. Augustin: Berater, Redner oder Teilnahme an klinischen Studien gesponsert durch Abbott/AbbVie, ALK Scherax, Almirall, Amgen, Beiersdorf, Biogen Idec, BMS, Boehringer Ingelheim, Celgene, Centocor, Dermira, Eli Lilly, Forward Pharma, Fresenius, Galderma, GSK, Hexal, Incyte, JanssenCilag, LEO Pharma, Lilly, Medac, Menlo, Merck, MSD Mylan, Novartis, Pfizer, Regeneron, Sandoz, SanofiAventis, Stallergenes, Stiefel, Teva, TK, Trevi, UCB und
Xenoport. F. Behrens: Forschungsförderung durch: Pfizer, Janssen, Chugai, Celgene und Roche; Berater und Referentenhonorar von: Pfizer, AbbVie, Sanofi, Lilly, Novartis, Genzyme, Boehringer, Janssen, MSD, Celgene, Roche, Chugai, BMS und UCB Pharma. S. Gerdes: Berater, Redner oder Teilnahme an klinischen Studien gesponsert durch AbbVie, Affibody AB, Akari Therapeutics PIc, Almirall-Hermal, Amgen, AnaptysBio, Argenx BV, AstraZeneca, Biogen Idec, Bioskin, BristolMyers Squibb, Boehringer-Ingelheim, Celgene, Centocor, Dermira, Eli Lilly, Foamix, Forward Pharma, Galderma, Hexal AG, Incyte Inc., Janssen-Cilag, Johnson \& Johnson, Kymab, Leo Pharma, Medac, MSD, Neubourg Skin Care GmbH, Novartis, Pfizer, Principia Biopharma, Regeneron, Sandoz, Sanofi-Aventis, Sienna Biopharmaceuticals, Takeda, Trevi Therapeutics, UCB und Vascular Biogenics. R. von Kiedrowski: Erbringt durch seine Dienstleistungsfirma $\mathrm{CMS}^{3} \mathrm{GmbH}$ Beratungsleistungen, Registerforschung, Tätigkeiten als Prüfarzt bei interventionellen und nichtinterventionellen Studien, sonstige Dienstleistungen und wissenschaftliche Vorträge für AbbVie, ALK Scherax, Almirall Hermal, Beiersdorf Dermo Medical, Biofrontera, Biogen, BMS, Boehringer Ingelheim, Celgene, Dermapharm, Foamix, Gilead, Hexal, Janssen-Cilag, Leo, Lilly, Meda, Medac, Menlo, MSD, Novartis, Dr. R. Pfleger, Pfizer, Regeneron, Sanofi, Stallergenes, Stiefel GSK, Tigercut und UCB. K. Schäkel: Berater, Redner oder Teilnahme an klinischen Studien gesponsert durch $\mathrm{AbbVie}, \mathrm{Am}$ gen, Almirall, Biogen, Boehringer Ingelheim, BristolMyers Squibb, Celgene, Chugai, Galderma, Janssen-Cilag, LEO Pharma, Lilly, Merck Sharp \& Dohme, Novartis, Pfizer, Polichem, Regeneron und UCB. M. Sticherling: Berater, Redner oder Teilnahme an klinischen Studien gesponsert durch Abbott/AbbVie, Almirall-Hermal, Amgen, Biogen Idec, Bioskin, Boehringer-Ingelheim, Celgene, Centocor, Eli Lilly, Galderma, Hexal AG, Incyte Inc., Janssen-Cilag, Johnson \& Johnson, Leo Pharma, Merck Serono, MSD, Novartis, Pfizer, Principia Biopharma, Regeneron, Sandoz, Sanofi-Aventis, ScheringPlough, Taked und UCB. D. Wilsmann-Theis: Berater, Redner oder Teilnahme an klinischen Studien gesponsert durch AbbVie, Almirall, Amgen, Anaptys, Biogen, Boehringer Ingelheim Pharma, Bristol Myers Squibb, Celgene, Forward Pharma, GlaxoSmithKline, Janssen-Cilag, Leo, Lilly, Medac, Merck Sharp \& Dohme Corp., Novartis, Pfizer, UCB und VBL. J. Wohlrab: Berater, Redner, Forschungsförderung oder Teilnahme an klinischen Studien gesponsert durch AbbVie, Almirall, Amgen, Biogen, Celgene, Galderma, GSK, Hexal, Janssen-Cilag, Leo, Lilly, MSD, Medac, Mylan, Novartis, Pfizer, Sanofi und UCB. J.-C. Simon: Berater, Redne oder Teilnahme an klinischen Studien gesponsert durch AbbVie, Amgen, Almirall, Bristol-Myers Squibb, Celgene, Galderma, Janssen-Cilag, LEO Pharma, Lilly, Merck Sharp\& Dohme, Novartis, Pfizer, Regeneron und $\mathrm{UCB}$

Für diesen Beitrag wurden von den Autoren keine Studien an Menschen oder Tieren durchgeführt. Für die aufgeführten Studien gelten die jeweils dort angegebenen ethischen Richtlinien.

Open Access. Dieser Artikel wird unter der Creative Commons Namensnennung 4.0 International Lizenz veröffentlicht, welche die Nutzung, Vervielfältigung, Bearbeitung, Verbreitung und Wiedergabe in jeglichem Medium und Format erlaubt, sofern Sie den/die ursprünglichen Autor(en) und die Quelle ordnungsgemäß nennen, einen Link zur Creative Commons Lizenz beifügen und angeben, ob Änderungen vorgenommen wurden. 
Die in diesem Artikel enthaltenen Bilder und sonstiges Drittmaterial unterliegen ebenfalls der genannten Creative Commons Lizenz, sofern sich aus der Abbildungslegende nichts anderes ergibt. Sofern das betreffende Material nicht unter der genannten Creative Commons Lizenz steht und die betreffende Handlung nicht nach gesetzlichen Vorschriften erlaubt ist, ist für die oben aufgeführten Weiterverwendungen des $\mathrm{Ma}$ terials die Einwilligung des jeweiligen Rechteinhabers einzuholen.

Weitere Details zur Lizenz entnehmen Sie bitte der Lizenzinformation auf http://creativecommons.org/ licenses/by/4.0/deed.de.

\section{Literatur}

1. Augustin $M$, Enk $A$, von Kiedrowski $R$ et al (2017) Einsatz von Systemtherapeutika und Biologika in der leitliniengerechten Therapie der mittelschweren bis schweren Psoriasis vulgaris. Psonet Mag 4:3-27

2. Augustin M, von Kiedrowski R, Körber A et al (2020) Verfahrensweise beider Systemtherapie von Patienten mit Psoriasis während der pandemischen Phase von SARS-CoV-2(Coronavirus). PsoNet

3. Bagel J, Duffin KC, Moore A et al (2017) The effect of secukinumab on moderate-to-severe scalp psoriasis: results of a 24-week, randomized, double-blind, placebo-controlled phase 3b study. JAm Acad Dermatol 77:667-674

4. Bakkour W, Purssell H, Chinoy H et al (2016) The risk of post-operative complications in psoriasis and psoriatic arthritis patients on biologic therapy undergoing surgical procedures. J Eur Acad Dermatol Venereol 30:86-91

5. Chioato A, Noseda E, Stevens M et al (2012) Treatment with the interleukin-17A-blocking antibody secukinumab does not interfere with the efficacy of influenza and meningococcal vaccinations in healthy subjects: results of an open-label, parallel-group, randomized singlecenter study. Clin Vaccine Immunol 19:1597-1602

6. Choi YM, Debbaneh M, Weinberg JM et al (2016) From the medical board of the national psoriasis foundation: perioperative management of systemic immunomodulatory agents in patients with psoriasis and psoriatic arthritis. J Am Acad Dermatol 75:798-805.e7

7. Deodhar A, Mcinnes I, Baraliakos X et al (2020) Secukinumab demonstrates a consistent safety profile in patients with psoriasis, psoriatic arthritis and ankylosing spondylitis over long term: Updated pooled safety analyses. Ann Rheum Dis 79:718

8. Deodhar A, Mease PJ, Mcinnes IB et al (2019) Longterm safety of secukinumab in patients with moderate-to-severe plaque psoriasis, psoriatic arthritis, and ankylosing spondylitis: integrated pooled clinical trial and post-marketing surveillance data. Arthritis Res Ther 21:111

9. Elnabawi YA, Dey AK, Goyal A et al (2019) Coronary artery plaque characteristics and treatment with biologic therapy in severe psoriasis: results from a prospective observational study. Cardiovasc Res 115:721-728

10. Fachinformation Secukinumab, Stand Dezember 2020

11. Furer V, Zisman D, Kaufman I et al (2020) Immunogenicity and safety of vaccination against seasonal influenza vaccine in patients with psoriatic arthritis treated with secukinumab. Vaccine 38:847-851

\section{Treatment of psoriasis with secukinumab. Practical guidance}

Background: Moderate to severe plaque psoriasis can be treated effectively with immunomodulating biologicals such as the interleukin-17A inhibitor secukinumab. In practice, however, questions often arise as to how to proceed in special situations, such as infections, comorbidity, pregnancy, or surgery.

Objectives: To address frequent questions about the treatment of plaque psoriasis with secukinumab in a consensus document of German psoriasis experts that supplements current guidelines.

Methods: In a virtual expert meeting in May 2020, practical aspects of the treatment of psoriasis were discussed based on the experience of the participants and on current literature. The results of this discussion were summarized in the present consensus document.

Results: This article provides practical guidance on case history, documentation of previous therapies, severity of psoriasis, and comorbidities before starting therapy with secukinumab. For patients treated with secukinumab, the course of action in case of vaccinations, chronic or acute infections, surgical interventions, special manifestations of psoriasis, and comorbidities including history of cancer and autoimmune disorders is discussed. Questions regarding family planning and health policy regulations are also addressed.

Discussion: The recommendations for the treatment of psoriasis with secukinumab summarized in this consensus document may contribute to achieve optimal therapy for patients and to improve their quality of life.

\section{Keywords}

Infections · Comorbidity · Perioperative management · Biological products · Special situations

12. Gottlieb A, Sullivan J, Van Doorn M et al (2017) Secukinumab shows significant efficacy in palmoplantar psoriasis: results from GESTURE, a randomized controlled trial. J Am Acad Dermatol 76:70-80

13. Gottlieb AB, Kubanov A, Van Doorn M et al (2020) Sustained efficacy of secukinumab in patients with moderate-to-severe palmoplantar psoriasis: 2.5year results from GESTURE, a randomized, doubleblind, placebo-controlled trial. Br J Dermatol 182:889-899

14. Imafuku S, Honma M, Okubo $Y$ et al (2016) Efficacy and safety of secukinumab in patients with generalized pustular psoriasis: a 52-week analysis from phase III open-label multicenter Japanese study. JDermatol 43:1011-1017

15. Mcinnes IB, Behrens F, Mease PJ et al (2020) Secukinumab versus adalimumab for treatment of active psoriatic arthritis (EXCEED): a double-blind, parallel-group, randomised, active-controlled, phase 3b trial. Lancet 395:1496-1505

16. Mrowietz U, Bachelez H, Burden AD et al (2019) Secukinumab for moderate-to-severe palmoplantar pustular psoriasis: results of the 2PRECISE study. J Am Acad Dermatol 80:1344-1352

17. Mrowietz U, Kragballe K, Reich K et al (2011) Definition of treatment goals for moderate to severe psoriasis: a European consensus. Arch Dermatol Res 303:1-10

18. Nast A, Altenburg A, Augustin M et al (2021) Deutsche S3-Leitlinie zur Therapie der Psoriasis vulgaris, adaptiert von EuroGuiDerm. J Dtsch Dermatol Ges (in print)

19. Nast A, Spuls PI, van der Kraaij G et al (2017) European S3-Guideline on the systemic treatment of psoriasis vulgaris-update apremilast and secukinumab-EDF in cooperation with EADV and IPC. JEur Acad Dermatol Venereol 31:1951-1963
20. Radtke MA, Mrowietz U, Feuerhahn J et al (2015) Früherkennung der Komorbidität bei Psoriasis: Konsensusempfehlungen der Nationalen KonferenzzurVersorgung der Psoriasis. JDtsch Dermatol Ges 13:674-690

21. Reich K, Sullivan J, Arenberger P et al (2020) Secukinumab shows high and sustained efficacy in nail psoriasis: 2.5 -year results from the randomized placebo-controlled TRANSFIGURE study. Br J Dermatol.https://doi.org/10.1111/bjd.19262

22. Reich K, Sullivan J, Arenberger P et al (2019) Effect of secukinumab on the clinical activity and disease burden of nail psoriasis: 32-week results from the randomized placebo-controlled TRANSFIGURE trial. Br J Dermatol 181:954-966

23. Sacchelli L, Patrizi A, Loi C et al (2019) Combination therapy of apremilast and secukinumab in patients with moderate-to-severe, recalcitrant plaque psoriasis. Clin Exp Dermatol 44:e243-e244

24. Schaberg T, Bauer T, Brinkmann F et al (2017) S2k Leitlinie:Tuberkulose im Erwachsenenalter

25. Ständige Impfkommission (2020) Empfehlungen der Ständigen Impfkommission (STIKO) am Robert Koch-Institut 2020/2021. Epidemiol Bull 34:1-68

26. Vaengebjerg S, Skov L, Egeberg A et al (2020) Prevalence, incidence, and risk of cancer in patients with psoriasis and psoriatic arthritis: a systematic review and meta-analysis. JAMA Dermatol 156:421-429

27. Von Kiedrowski R, Dirschka T, Kurzen Het al (2019) Empfehlungen für die ambulante Versorgung von Psoriasis vulgaris - Aktualisierter praxisnaher Behandlungspfad. Dermatologe 09(Suppl):1-24

28. Von Stebut E, Boehncke WH, Ghoreschi K et al (2019) IL-17A in psoriasis and beyond: cardiovascular and metabolic implications. Front Immunol 10:3096 
29. Von Stebut E, Reich K, Thaçi D et al (2019) Impact of secukinumab on endothelial dysfunction and other cardiovascular disease parameters in psoriasis patients over 52 weeks. J Invest Dermatol 139:1054-1062

30. Wagner N, Assmus F, Arendt G et al (2019) Impfen beilmmundefizienz. Anwendungshinweisezu den von der Ständigen Impfkommission empfohlenen Impfungen. (IV) Impfen bei Autoimmunkrankheiten, bei anderen chronisch-entzündlichen Erkrankungen und unter immunmodulatorischer Therapie. Bundesgesundheitsblatt Gesundheitsforschung Gesundheitsschutz 62:494-515

31. Warren RB, Reich K, Langley RG et al (2018) Secukinumab in pregnancy: outcomes in psoriasis, psoriatic arthritis and ankylosing spondylitis from the global safety database. Br J Dermatol 179:1205-1207

32. Wohlrab J, Fiedler G, Gerdes S et al (2013) Recommendations for detection of individual risk for comorbidities in patients with psoriasis. Arch Dermatol Res 305:91-98

\section{Möchten Sie einen Beitrag für die Zeitschrift Der Hautarzt einreichen?}

Wir freuen uns, dass Sie unsere Zeitschrift Der Hautarzt mitgestalten möchten. Um Ihnen bei der Manuskripterstellung behilflich zu sein, haben wir für unsere Autor*innen Hinweise zusammen gestellt, die Sie im Internet finden unter www.DerHautarzt.de (For Authors).

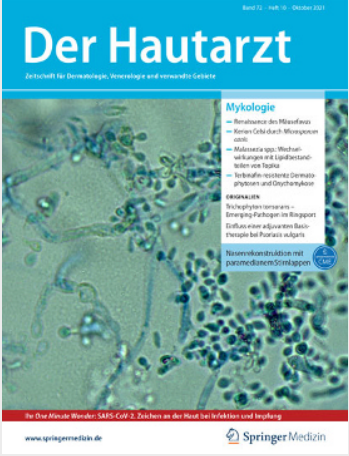

Bitte senden Sie Ihren fertigen Beitrag an:

\section{Originalien/Übersichten/}

Kasuistiken/Wie lautet Ihre Diagnose?

Prof. Dr. Daniela Bruch-Gerharz

Hautklinik des Universitätsklinikums

Düsseldorf, Moorenstr. 5,

40225 Düsseldorf

sdgehrke@googlemail.com

Dermatoskopie - Fall des Monats:

Prof. Dr. Andreas Blum

Hautarztpraxis Konstanz und Lehrpraxis der Medizinischen Fakultät Universität Tübingen Augustinerplatz 7

78462 Konstanz

a.blum@derma.de

\section{Rezepturtipp für die Praxis aus der} Praxis:

Bitte schreiben Sie bei Fragen an:

hautarzt@dac-nrf.de

\section{One Minute Wonder:}

PD Dr. Dr. Alexander Zink, Dr. Sarah Preis Klinik und Poliklinik für Dermatologie und Allergologie am Biederstein,

TU München

Bitte schreiben Sie bei Fragen an

die Verlagsredaktion:

julie.kind@springer.com

\section{CME Zertifizierte Fortbildung:}

Anfragen an:

Prof. Dr. Evelyn Gaffal

Universitätshautklinik

Universitätsklinikum Magdeburg

Labor für Experimentelle Dermatologie

Leipziger Straße 44

39120 Magdeburg

evelyn.gaffal@med.ovgu.de

Prof. Dr. Sonja Ständer

Klinik und Poliklinik für Hautkrankheiten,

Universitätsklinikum Münster

Von-Esmarch-Str. 58

48149 Münster

sonja.staender@uni-muenster.de

Prof. Dr. Rolf-Markus Szeimies

Klinik für Dermatologie und Allergologie, Knappschaftskrankenhaus Recklinghausen, Klinikum Vest GmbH,

Dorstener Str. 151,

45657 Recklinghausen

dermatologie@kk-recklinghausen.de

PD Dr. Dr. Alexander Zink

Klinik und Poliklinik für Dermatologie und

Allergologie am Biederstein,

Technische Universität München

Biedersteiner Str. 29

80802 München

alexander.zink@tum.de 